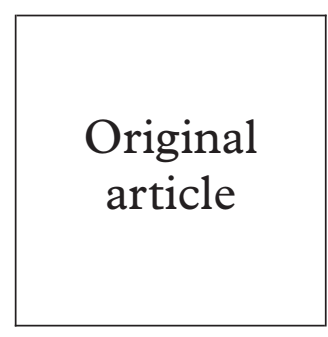

\title{
A study of anal intraepithelial neoplasia in HIV positive homosexual men
}

\author{
H B Lacey, G E Wilson, P Tilston, E G L Wilkins, A S Bailey, G Corbitt, P M Green
}

\begin{abstract}
Objectives: To determine the prevalence of high grade anal intraepithelial neoplasia (HGAIN), the value of anal cytology in screening for HGAIN, and the characterisation of epidemiological factors and human papillomavirus (HPV) types.

Methods: Prospective cohort study of HIV positive homosexual men. Subjects were interviewed, underwent STD, anal cytological, and HPV screening at enrolment and at subsequent follow up visits with anoscopy and biopsy at the final visit. 57 enrolled, average CD4 count $273 \times 10^{6} / 1(10-$ 588); 41 completed the cytological surveillance over the follow up period (181 visits, average follow up 17 months), 38 of these had anoscopy and anal biopsy.

Results: Oncogenic HPV types were detected in $84 \%$ and high grade dyskaryosis in $10.5 \%$ (6/57) at enrolment. There was a $70 \%$ incidence of high grade dyskaryosis during follow up in patients with negative/warty or low grade dyskaryosis at enrolment. Anoscopy correlated with histology in high grade AIN lesions (sensitivity $91 \%$, specificity $54 \%$ ) and cytology was $78 \%$ sensitive $(18 / 23)$ for HGAIN on biopsy.

Conclusions: AIN and infection with multiple oncogenic HPV types are very common among immunosuppressed HIV positive homosexual men. Apparent progression from low to high grade cytological changes occurred over a short follow up period, with no cases of carcinoma. All 23 cases of HGAIN were predicted by cytology and/or anoscopy. Future studies focusing on the risk of progression to carcinoma are needed before applying anal cytology as a screening tool for AIN in this population.
\end{abstract}

(Sex Transm Inf 1999;75:172-177)

Keywords: anal intraepithelial neoplasia; HIV; human papillomavirus

\section{Introduction}

The development of both cervical and anal intraepithelial neoplasia (CIN, AIN) is strongly associated with human papillomavirus (HPV) infection..$^{1-3}$ The high risk HPV types 16 and 18 are also frequently found in invasive carcinoma tissue from both these anatomical sites. ${ }^{4-6}$ Anal intraepithelial lesions (squamous cell dyskaryosis and AIN) are common in homosexual men with increased risk associated with HIV seropositivity, advancing immunosuppression (associated with declining CD4 count), the presence of high risk oncogenic HPV infection, and epidemiological factors such as smoking. ${ }^{7-9} \mathrm{HPV}$ infection appears to run a more aggressive course with increasing immunodeficiency ${ }^{10}$ and although an AIDS diagnosis carries a greatly increased relative risk for the development of anal cancer ${ }^{11}$ it appears that much of this risk is attributable to the risk factor of anoreceptive sex with HIV associated immunosuppression conferring a further small additional risk. ${ }^{12}$

A recent prospective study of HIV positive homosexual men found a $4.9 \%$ prevalence of high grade anal disease at baseline (17/346) and during a 4 year follow up, $34 \%$ of 277 subjects who, at baseline cytological and anoscopic assessment, were classified as having low grade or benign anal disease developed biopsy proved high grade disease. Comparable figures in the HIV negative control group were $0.4 \%$ baseline prevalence of high grade disease with a $13 \%$ progression rate. ${ }^{7}$ Other studies have found an incidence of high grade disease of $15-16 \%$ over 17 months to 3 years of follow up. ${ }^{813}$ As high grade intraepithelial lesions are the precursor of most cervical carcinomas the similarities between CIN and AIN lesions together with the reported rise in incidence of anal cancer in the populations at risk of AIN suggest that high grade AIN may be the precursor of invasive carcinoma. Whereas the natural history and malignant potential of CIN lesions have been well described, ${ }^{14}{ }^{15}$ the temporal relation between the development of HPV infection, AIN, and anal cancer is currently poorly defined.

The aim of this study was to determine the prevalence and types of anal HPV infection in a cohort of male homosexuals with HIV infection, to assess progression of lesions on cytology over time, to examine the sensitivity and specificity of cytology in the detection of high grade disease, and to examine the natural history of anal epithelial abnormality in this group in relation to HPV infection, HIV mediated immunosuppression, and other epidemiological factors.

\section{Materials and methods}

STUDY POPULATION AND DESIGN

In January 1994 HIV positive homosexual men were recruited for this study. Subjects were recruited from a larger cohort (300 patients, $33 \%$ CDC group C) attending North Manchester General Hospital for their HIV care. Patients were given an information sheet describing the project and were asked if they wished to volunteer. The project was approved by the ethics committee and over the following 
18 months, 57 patients were enrolled after written consent was obtained. Subjects were interviewed about demographic characteristics, sexual behaviour, and history of sexually transmitted diseases (STDs). The physical examination included an STD screen. Anal cytological specimens were taken by visualising the lower anal canal with a proctoscope and using a Dacron swab to sample the lower anal canal from the area of the squamocolumnar junction to the anal margin. The swab was rolled onto a labelled glass slide and immediately sprayed with Cytofix (Cell Path) cytological fixative. The same swab was then placed into $1 \mathrm{ml}$ phosphate buffered saline (PBS) containing benzylpenicillin (100 units $/ \mathrm{ml}$ ), streptomycin $(100 \mu \mathrm{g} / \mathrm{ml})$ and amphotericin B $(2.5 \mu \mathrm{g} / \mathrm{ml})$ and stored at $-20^{\circ} \mathrm{C}$ before HPV analysis. The results of regular CD4 subset lymphocyte counts were available from each subject's hospital notes. At each 4 monthly follow up visit information relating to anal symptoms, antiretroviral drug therapy, and new sexual partners was requested. Further STD tests were taken only if indicated. The perianal area was then examined and specimens taken for anal cytology and HPV analysis.

ANOSCOPY AND BIOPSY

At the final follow up visit subjects were given $2 \%$ lignocaine gel to insert into the anal canal before anoscopy. All anoscopy was performed by one physician (HBL), using an Olympus colposcope. The perianal area was inspected before and after the application of $5 \%$ acetic acid. A transparent proctoscope was inserted and Dacron swab used to take a cytological and HPV specimen. The proctoscope was then reinserted and the anal canal inspected before and after application of 5\% acetic acid. Using colposcopic criteria standardised for the cervix, anoscopic appearances were graded as low grade changes (mild aceto-whitening with or without fine punctation/mosaicism) or high grade (dense aceto-whitening, coarse punctation, gland cuffing, and/or coarse mosaicism) changes. The highest grade of epithelial abnormality was selected and infiltrated with $2 \%$ xylocaine with 1:80 000 adrenaline using a dental syringe before taking a biopsy using Eppendorfer colposcopic biopsy forceps. The specimen was fixed in $10 \%$ neutral buffered formalin and sent for histological examination.

CYTOLOGICAL AND HISTOLOGICAL EXAMINATIONS Fixed cytological smears were stained according to the Papanicolaou method. All smears were examined by one observer (GEW) who had knowledge of the previous results but not of the clinical findings at the time when the smear was taken. The adequacy of the smear was assessed on the basis of the cellularity of the smear, the presence of columnar and/or metaplastic squamous cells from the anorectal junction, and acceptable fixation and staining of the smears. Evidence of HPV infection was based on the presence of koilocytosis, individual cell keratinisation, anucleate squamous cells, and multinucleated squamous cells. Squamous epithelial abnormalities, where present, were reported as high grade dyskaryosis (HGdyskaryosis $=$ moderate and severe dyskaryosis) or low grade epithelial abnormality (LGEA = warty features with borderline nuclear abnormality and/or mild dyskaryosis). Biopsy specimens were embedded in paraffin wax following fixation. Sections of $5 \mu \mathrm{m}$ were stained with haematoxylin and eosin and not less than three sections were examined from each biopsy. Histological criteria for the diagnosis of HPV-like changes and AIN were identical to those routinely used in the assessment of HPV-like changes and CIN in the cervix. AIN, when present, was graded as high grade AIN (HGAIN= AIN2 and AIN3) or low grade squamous intraepithelial lesions (LGSIL= warty features and/or AIN1).

HPV DNA DETECTION

Total DNA was extracted in lysis buffer $(10$ $\mathrm{mM}$ TRIS- $\mathrm{HCl} \mathrm{pH} 8.3,1 \mathrm{mM}$ EDTA, 0.5\% Triton-X-100, $0.001 \%$ SDS) containing 250 $\mu \mathrm{g} / \mathrm{ml}$ proteinase $\mathrm{K}$ at $56^{\circ} \mathrm{C}$ for 2 hours followed by boiling for 15 minutes, to inactivate the proteinase $\mathrm{K}$. Five $\mu$ l of lysate was first amplified with the B globin primers GH20 and $\mathrm{PC} 4^{16}$ in a $50 \mu \mathrm{l}$ reaction volume under the following conditions: $1 \times \mathrm{PCR}$ buffer $(67 \mathrm{mM}$ TRIS-HCl pH 8.4, $16 \mathrm{mM}$ ammonium sulphate, $2 \mathrm{mM} \mathrm{MgCl}, 0.1 \%$ gelatin), $220 \mu \mathrm{M}$ each deoxynucleotide triphosphate, $10 \mathrm{pmol}$ each primer, and 1 unit Taq DNA polymerase. Thermal cycling was performed over 50 cycles as follows: $94^{\circ} \mathrm{C}$ for 7 minutes, $62^{\circ} \mathrm{C}$ for 1 minute, $72^{\circ} \mathrm{C}$ for 1 minute $(1$ cycle $) ; 94^{\circ} \mathrm{C}$ for 1 minute, $62^{\circ} \mathrm{C}$ for 1 minute, $72^{\circ} \mathrm{C}$ for 1 minute (49 cycles). Amplified DNA was resolved in $1.5 \%$ agarose and visualised by ultraviolet illumination following ethidium bromide staining. Subject to a positive B globin result, a further $5 \mu$ of lysate was amplified using $50 \mathrm{pmol}$ of the consensus HPV primers GP5+/GP $6+{ }^{17}$ under the same conditions as above but utilising a $40^{\circ} \mathrm{C}$ annealing temperature.

Those specimens giving a positive result with GP5+/6+ were then subjected to HPV genotyping with type specific primers (10 pmol) for HPV types $6 / 11,16,18,31$, and $33 .{ }^{18-20}$ Thermal cycling was performed as described above utilising a $55^{\circ} \mathrm{C}$ annealing temperature and amplified DNA resolved in $2 \%$ agarose. The risk of contamination of specimens was minimised by the physical separation of all pre- and post-amplification steps in separate, dedicated laboratories and by the inclusion of appropriate negative controls in every run.

STUDY END POINT

The study end point was reached when the subject had completed a minimum of 14 months' follow up when anoscopy, cytology, and biopsy were performed.

\section{Results}

The mean age of the 57 men enrolled was 35 years (range 19-62). The average CD4 count was $273 \times 10^{6} / 1$ (range $10-588$ ). The prevalence of HGdyskaryosis at enrolment was 
Table 1 Cytology and CD4 count $\left(\times 10^{6} / l\right)$ results at enrolment (57 patients)

\begin{tabular}{|c|c|c|c|c|c|}
\hline \multirow[b]{2}{*}{$C D 4$} & \multicolumn{2}{|l|}{ Cytology results } & \multirow[b]{2}{*}{$L G E A$} & \multirow[b]{2}{*}{ HGdyskaryosis } & \multirow[b]{2}{*}{ Total } \\
\hline & Unsatisfactory & Negative & & & \\
\hline$<51$ & 2 & 2 & 3 & 2 & 9 \\
\hline $51-200$ & 1 & 6 & 6 & 1 & 14 \\
\hline $201-500$ & 1 & 9 & 14 & 3 & 27 \\
\hline$>500$ & 0 & 3 & 4 & 0 & 7 \\
\hline Total & 4 & 30 & 17 & 6 & 57 \\
\hline
\end{tabular}

HGdyskaryosis = moderate and/or severe dyskaryosis, LGEA = warty features with borderline nuclear abnormality and/or mild dyskaryosis.

Table 2 Initial, final, and most abnormal cytology results (41 patients)

\begin{tabular}{llll}
\hline & $\begin{array}{l}\text { First smear } \\
\text { No } \% \text { ) }\end{array}$ & $\begin{array}{l}\text { Last smear } \\
\text { No (\%) }\end{array}$ & $\begin{array}{l}\text { Most } \\
\text { abnormal } \\
\text { smear } \\
\text { No } \%\end{array}$ \\
\hline Unsatisfactory & $3(7)$ & 0 & 0 \\
Negative & $15(37)$ & $3(7)$ & $1(3)$ \\
LGEA & $19(46)$ & $14(34)$ & $10(24)$ \\
HGdyskaryosis & $4(10)$ & $24(59)$ & $30(73)$ \\
\hline
\end{tabular}

HGdyskaryosis = moderate and/or severe dyskaryosis, LGEA = warty features with borderline nuclear abnormality and or mild dyskaryosis.

Highly significant difference in the finding of HGdyskaryosis at enrolment and at the last smear, $\mathrm{p}=<0.0001 \mathrm{McNemar}$ 's test.

$10.5 \%(6 / 57)$. The results of cytology and CD4 counts at enrolment are shown in table 1.

Of the 57 men who enrolled in the study 41 completed the cytological follow up. There were eight deaths and eight declined to continue participating in the study after enrolment, eight had LGEA, two HGdyskaryosis, five negative, and one unsatisfactory cytology at enrolment. The baseline, final, and most abnormal cytology results on the 41 subjects who completed cytological follow up are shown in table 2. Second smears on the three with unsatisfactory initial smears showed two to have LGEA and one negative cytology. The results of sequential smears are represented in figure 1. By the end of the study period HGdyskaryosis had been detected on $73 \%$ (30/41) of subjects on at least one smear. In the $37 / 41$ with LGEA or negative initial smears there was a $70 \%$ incidence of HGdyskaryosis during follow up (26/37) with a 54\% apparent progression to HGdyskaryosis when comparing the first and final smear (20/37).

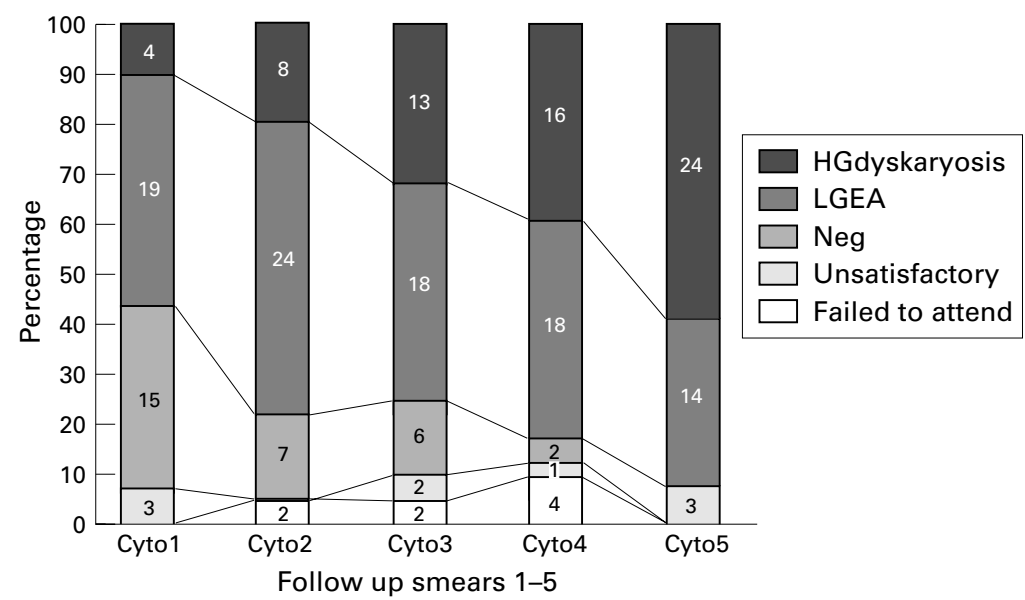

Figure 1 Results of sequential smears (41 patients). HGdyskaryosis = moderate and severe dyskaryosis, LGEA = warty features with borderline nuclear abnormality and or mild dyskaryosis, Neg = negative.
The study end point with anoscopy and biopsy was reached by 38 subjects (three of the 41 patients who completed the cytological follow up refused anoscopy and biopsy). The average follow up was 17 months (range 14-21 months, total 650 months); 184 outpatient visits were attended and 184 cytology and HPV specimens were available for analysis. The mean CD 4 count of the 38 study patients was 299 at enrolment and was 276 at completion of the study (mean change $22.3,95 \%$ confidence interval -7.7, 52.4). Although CD4 count decreased slightly during the follow up period this was not statistically significant (paired $t$ test $[\mathrm{t}(37)=1.5 ; \mathrm{p}=0.14])$.

CORRELATION OF CYTOLOGICAL, HISTOLOGICAL, AND ANOSCOPIC FINDINGS

One biopsy sample was inadequate for analysis and three showed AIN but accurate grading was not possible because of epithelial fragmentation. Results were available on $34 / 38$ subjects who had satisfactory anal biopsies. High grade AIN was detected in 23 subjects, 20 of whom had initial smears that were negative or showed LGEA giving an apparent progression rate of $66 \%(20 / 30)$ from LGEA/negative cytology at enrolment to HGAIN on histology. When comparing histology with cytology taken at the time of biopsy, the sensitivity of a single smear with HGdyskaryosis for detecting HGAIN was $78 \%$ (table 3) No patient was found to have invasive carcinoma on biopsy and no patient who had a negative smear at the time of biopsy was found to have high grade AIN. Cytology at the time of biopsy underestimated the grade of abnormality in five patients suggesting LGEA when biopsy found HGAIN; however, in four cases the histological abnormality had been predicted by a HG dyskaryotic smear earlier in the follow up period. The anoscopist was aware of the previous smear results. Some subjects were found to have predominantly perianal disease although most had some changes in the anal canal itself. These changes were often patchy and multifocal and were found anywhere from the squamocolumnar junction to and including the perianal region.

PREVALENCE AND TYPES OF HPV

Positive HPV results were found in 48 of the 57 patients that initially enrolled ( 37 having multiple HPV types); six were negative, two were untypable, and one specimen was unsatisfac-

Table 3 Correlation of final smear and anoscopic appearance with histology (34 patients)

\begin{tabular}{llll}
\hline & Histology & & \\
Final cytology & HGAIN & LGSIL & Negative \\
\hline HG dyskaryosis & 18 & 2 & 0 \\
LGEA & 5 & 8 & 0 \\
Negative & 0 & 0 & 1 \\
Total & 23 & 10 & 1 \\
Anoscopyt & HGAIN & LGSIL/Neg & \\
\hline HG changes & 21 & 5 & \\
LG/neg changes & 2 & 6 & \\
Total & 23 & 11 & \\
& & & \\
\hline
\end{tabular}

Sensitivity in detecting HGAIN.

$\star$ Sensitivity $78 \%$, specificity $82 \%$.

†Sensitivity $91 \%$, specificity $54 \%$. 


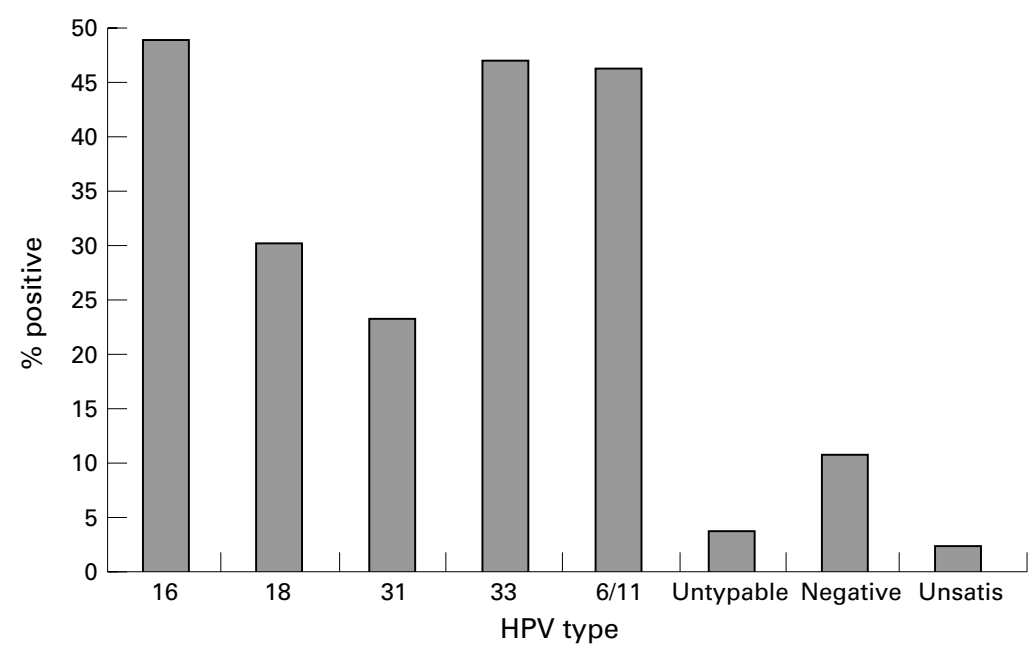

Figure 2 Distribution of HPV types at enrolment (57 patients).

tory. At least one high risk type was found in 46 $(81 \%)$ subjects. The distribution of HPV types is shown in figure 2 .

At enrolment 33 of the 38 subjects who completed the study were HPV positive, 24 of these having multiple types. Four of the five initially negative for HPV were found to be positive on subsequent specimens. Oncogenic types $(16,18,31$, and 33) were found at enrolment in 29/33 HPV positive subjects (two HPV 6/11 alone and two untypable HPVs). By the end of follow up oncogenic HPVs had been detected in 36 of the 38 subjects, one had persistently untypable HPV, and the one patient persistently negative for HPV was found to have HGdyskaryosis and HGAIN on biopsy. The same HPV type pattern persisted in 24 of the 38 who completed the follow up period, seven subjects lost one or more HPV types (on at least two sequential visits), and seven gained one or more HPV types. During the follow up period 22 subjects had new sexual partners. New STDs were diagnosed in seven subjects during the study (three cases of gonorrhoea, one non-specific urethritis, one chlamydia urethritis, one secondary syphilis, and one initial genital herpes).

\section{Discussion}

Despite the small sample size this study has shown a surprisingly high incidence of oncogenic HPV infection and anal cytological abnormalities. Our study population was small and may not be representative of HIV positive men as a whole. Most $(50 / 57$ CD 4 <500) of our study group were immunosuppressed to some degree and as lower CD4 count has been linked with increased risk of AIN this may have contributed to the high incidence of abnormalities including the high prevalence of HGdyskaryosis at enrolment $(10.5 \%)$.

There have been very few studies on anal neoplasia, ${ }^{3-9} 13^{21-23}$ and many are cross sectional. Comparison between the results of these studies is problematic. One difficulty has been the definition of AIN, with dyskaryosis on cytology without histological confirmation being defined as AIN in some cases. ${ }^{3813}$ The epithelial abnormalities in many studies have been predominantly low grade lesions and, as in the interpretation of cervical cytology, differentiation between the cytological features of HPV infection and low grade dyskaryosis can be difficult. As it is HGAIN that is more likely to be the precursor of invasive disease, a screening test must be able to differentiate this clinically significant disease from low grade disease which may just be related to HPV infection which is exceedingly common (93\%) in this group of patients. ${ }^{10}$

We had hoped by analysing epidemiological characteristics and HPV types to identify factors conferring increased risk of high grade disease but the presence of oncogenic HPV types in almost all patients has made this aspect impossible and as only one of our subjects had persistently negative smears we were not able to demonstrate any association between the grading of cytological abnormalities and behavioural and epidemiological factors. There was, however, one patient who had high grade abnormalities on both cytology and biopsy who was persistently HPV negative. This has been noted before ${ }^{7}$ and may indicate development of HGAIN in the absence of HPV or the inability to detect some HPVs with current PCR technology.

Two HIV positive cohorts have previously been followed prospectively. ${ }^{7822}$ Palefsky et al ${ }^{7}$ found $38 \%(106 / 277)$ of those who had negative or low grade epithelial abnormalities at enrolment progressed to high grade disease (as defined by histology in $88 \%$ or cytology in $12 \%$ ) over a follow up period of 4 years. Critchlow et $a l^{8}$ showed, over 20 months of follow up, progression to high grade disease on cytology or histology in $15 \%(24 / 158)$ who had negative cytology and anoscopy at baseline. Our results show what appears to be a higher rate of progression to high grade epithelial abnormalities (54\% on cytology and $66 \%$ to HGAIN on histology) in those with negative or low grade abnormalities on cytology at baseline. Although striking, it must be emphasised that these results must be interpreted with caution for a number of reasons.

Critchlow $^{8}$ and Palefsky ${ }^{7}$ took biopsies at baseline only from those who had cytological and or colposcopic abnormalities. We omitted all biopsies at enrolment as we felt this would lead to recruitment difficulties and might bias the reporting of cytology specimens as the histopathologist was not blinded to previous results. As histology was omitted, cytology probably underestimated, to some extent, the baseline prevalence of high grade disease. As there was little change in CD4 count during our study, advancing immunosuppression did not appear to account for the increase in detection of HGdyskaryosis. Interobserver variation in cervical cytology reporting is well documented $^{24}$ and although there are no similar studies of anal cytology reporting it is likely that similar differences also exist making comparisons between studies subject to variation in human interpretation. The increased detection over time of high grade disease in our study may, in part, be due to increasing experience of the histopathologist who was not blinded to previous results. Although the apparent pro- 
gression was perhaps not as large as the results indicate for the reasons above we have, however, shown better correlation between histology and cytology in high grade disease than previous studies which used a blind cytology sampling technique on a population with a lower prevalence of high grade AIN. ${ }^{21}{ }^{22}$

In validating the anal cytology we have assumed that histology is the gold standard for diagnosing or excluding AIN. There is, however, interobserver variation in the interpretation of histology which produces inherent inaccuracies which are difficult to control and affect comparison with other studies. ${ }^{25}$ Anal cytology has been found to be of variable sensitivity in the detection of AIN. One cross sectional study of 56 men found the sensitivity of a single smear showing any degree of AIN to be $34 \%$ (specificity $72 \%$ ) in detecting any grade of AIN on histology. Sensitivity in detection of high grade disease by cytology was even lower $(14 \%, 3 / 22)$ but numbers were very small. ${ }^{21} \mathrm{~A}$ much larger study found the sensitivity of cytology in the detection of AIN of any grade in HIV positive men to be $46 \%$ (46/100) rising to $56 \%$ when data from subsequent follow up visits were included. There was, however, a relatively poor correlation $(27 \%, 3 / 11)$ between histology and cytology for high grade disease. ${ }^{22}$ Our results indicate the sensitivity of a single smear in the detection of HGAIN to be $78 \%$. While the specificity was only $83 \%$ this may have less importance in a high prevalence population.

Of the 23 patients with HGAIN on biopsy in this study, 22 were predicted by at least one follow up smear showing HGdyskaryosis. The one patient, who had HGAIN on biopsy in whom smears persistently underestimated the abnormality had, on anoscopy, almost exclusively perianal disease which may have been missed on cytology. In previous similar studies $^{21} 22$ cytology/histology mismatches have occurred (this is also well recognised in colposcopy clinics). Detection of disease by anoscopy is also subject to interobserver variation. The epithelial abnormalities inside the anal canal were often patchy and the anatomy of the anal canal makes biopsy sampling error a likely explanation for the four cases where cytology had suggested high grade changes that were not found on biopsy. All those patients who had high grade AIN in the anal canal on biopsy were found at some stage during the follow up period to have had a high grade dyskaryotic smear. A combination of serial smears and anoscopy detected all those patients with high grade AIN as anoscopy detected the patient with isolated perianal HGAIN.

While our results need to be interpreted with care because of the small numbers it appears that anoscopy and cytology could be a useful screening procedure in the detection of high grade disease in immunosuppressed HIV positive homosexual men.

Although data from the United States indicate increased risk of anal cancer among homosexual men the numbers of cases are still very low. Despite the high incidence of HGAIN in our cohort we have found no cases of anal carcinoma. We now have data from 55 patient years of follow up in those with HGAIN on biopsy and have not yet identified any carcinomas (unpublished data). Analogies have been drawn with CIN which has an increased incidence in HIV positive women. ${ }^{26}$ There is, however, no evidence of an increased progression to carcinoma in HIV infected women and at present the risk of progression from high grade AIN to anal carcinoma in men is unknown. There is no current consensus about whether patients with high grade AIN should be treated and although ablative treatments have been suggested for $\mathrm{HGAIN}^{27}$ there are no published results of the efficacy of ablative or laser treatment in these patients. HIV positive women have a higher recurrence rate of CIN after treatment than HIV negative women ${ }^{28}$ and it is likely that this will also be the case in treatment of anal disease which is likely to result in more side effects than cervical treatment. While questions remain to be answered about the significance of high grade AIN in this group of patients it seems wise to urge caution when considering screening programmes until more data about the natural history and efficacy of treatment options are known.

We are grateful for the help and advice of Dr Cynthia Stanbridge, consultant cytopathologist at Central Manchester Healthcare NHS Trust.

Contributors: HB Lacey, GE Wilson, EGL Wilkins, and G Contributors: Here involved in the study design. EL Wilkins and HB Corbitt were involved in the study design. EL Wilkins and HB Tilston, and GE Wilson contributed to the final manuscript and HB Lacey, GE Wilson, AS Bailey, P Tilston, G Corbitt, an PM Green were involved in the data collection and processing. Funding: Grant support RL Gardner Cancer Research Fund. Conflict of interest: none.

1 Kjaer SK, van den Brule AJ, Bock JE, et al. Human papillomavirus - the most significant risk determinant of cervical intraepithelial neoplasia. Int f Cancer 1996;66:601-

2 Rozendaal L, Walboomers JM, van der Linden JC, et al. PCR-based high-risk HPV test in cervical cancer screening gives objective risk assessment of women with cytomorphologically normal cervical smears. Int $\mathcal{F}$ Cancer 1996;68:766-

3 Palefsky JM, Gonzales J, Greenblatt RM, et al. Anal intraepithelial neoplasia and anal papillomavirus infection among homosexual males with group IV HIV disease. FAMA 1990;263:2911-16.

4 van den Brule AJC, Snijders PJF, Gordun R, et al. General primer-mediated polymerase chain reaction permits the detection of sequenced and still unsequenced human papillomavirus genotypes in cervical scrapes and carcinomas Int $\mathcal{F}$ Cancer 1990;45:644-9.

5 Zaki SR, Judd R, Coffield LM, et al. Human papillomavirus infection and anal carcinoma. Retrospective analysis by in situ hybridisation and the polymerase chain reaction. $A m \mathcal{F}$ Pathol 1992;140:1345-55.

6 Palefsky JM, Holly EA, Gonzales J, et al. Detection of human papillomavirus DNA in anal intraepithelial neoplasia and anal cancer. Cancer Res 1991;51:1014-19.

7 Palefsky JM, Holly EA, Ralston ML, et al. High incidence of high grade squamous intra-epithelial lesions among high grade squamous intra-epithelial lesions among HIV-positive and HIV-negative

8 Critchlow CW, Surawicz CM, Holmes KK, et al. Prospective study of high grade anal squamous intraepithelial neoplasia in a cohort of homosexual men: influence of HIV infection, immunosuppression and human papillomavirus infection. AIDS 1995;9:1255-62.

9 Palefsky JM, Shiboski S, Moss A. Risk factors for anal human papillomavirus infection and anal cytologic abnormalities in HIV positive and HIV negative homosexual men. F Acquir Immune Defic Syndr 1994;7:599-606.

10 Palefsky JM, Holly EA, Ralston ML, et al. Prevalence and risk factors for human papillomavirus infection of the anal risk factors for human papilomavirus infection of the anal and HIV negative homosexual men. F Infect Dis 1998;177: 361-7.

11 Melbye M, Cote TR, Kessler L, et al and the AIDS/Cancer Working Group. High incidence of anal cancer among AIDS patients. Lancet 1994;343:636-9. 
12 Goedert JJ, Cote TR, Virgo P, et al. Spectrum of AIDS-associated malignant disorders. Lancet 1998;351: 1833-9.

13 Palefsky JM, Holly EA, Gonzales J, et al. Natural history of anal cytological abnormalities and papillomavirus infection among homosexual men with group IV HIV disease. $\mathcal{F}$ Acquir Immune Defic Syndr 1992;5:1258-65.

14 Ostor AG. Natural history of cervical intraepithelial neoplasia: a critical view. Int F Gynecol Pathol 1993;12:18692.

15 Syrjanen KJ. Spontaneous evolution of intraepithelia lesions according to the grade and type of the implicated human papillomavirus (HPV). Eur f Obstet Gynecol Reprod Biol 1996;65:45-53.

16 Bauer HM, Greer CE, Manos MM. Determination of genital human papillomavirus infection by consensus PCR amplification. In: Herrington CS, McGee J, eds. Diagnostic molecular pathology. A practical approach. Oxford: Oxford

17 de Roda Husman AM, Walboomers JMM, van den Brule AJC, et al. The use of general primers GP5 and GP6 elongated at their 3 ends with adjacent highly conserved sequences improves human papillomavirus detection by PCR. ₹ Gen Virol 1995;76:1057-62.

18 Soler C, Allivert P, Chardonnet Y, et al. Detection of human papillomavirus types $6,11,16$ and 18 in mucosal and cutaneous lesions by the multiplex polymerase chain reaction. $\mathcal{F}$ Virol Methods 1991;35:143-57.

19 van den Brule AJC, Meijer CJLM, Bakels V, et al. Rapid detection of human papillomavirus in cervical scrapes by combined general primer-mediated and type-specific polymerase chain reaction. 7 Clin Microbiol 1990;28:2739 43.
20 Vandenvelde C, Scheen R, Van Pachterbeke C, et al. Prevalence of high risk genital papillomaviruses in the Belgian female population determined by fast multiplex polymerase chain reaction. $\mathcal{F}$ Med Virol 1992;36:279-82.

21 de Ruiter A, Carter P, Katz D.R, et al. A comparison between cytology and histology to detect anal intraepithelial neoplasia. Genitourin Med 1994;70:22-5.

22 Palefsky JM, Holly EA, Hogeboom CJ, et al. Anal cytology as a screening tool for anal squamous intraepithelial lesions. F Acq Immun Defic Syndr 1997;14:415-22.

23 Carter PS, de Ruiter A, Whatrup C, et al. Human immunodeficiency virus infection and genital warts as risk factors for anal intraepithelial neoplasia in homosexual men. $B r f$ Surg 1995;82:473-4.

24 Sherman ME, Schiffman MH, Erozan YS, et al. The Bethesda system, a proposal for reporting abnormal cerviBethesda system, a proposal for reporting abnormal cervi-
cal smears based on the reproducibility of cytopathologic cal smears based on the reproducibility of cytop

25 Carter PS, Sheffield JP, Shepherd DH, et al. Interobserver variation in the reporting of the histopathological grading of anal intraepithelial neoplasia. F Clin Pathol 1994;47: 1032-4.

26 Vermund SH, Kelley KF, Klein RS, et al. High risk of human papillomavirus infection and cervical squamous intraepithelial lesions among women with symptomatic human immunodeficiency virus infection. Am 7 Obstet Gynecol 1991;165:392-400.

27 Northfelt DW, Swift PS, Palefsky JM. Anal neoplasia, pathogenesis, diagnosis and management. Haematol Oncol plin NAm 1996;10:1177-87.

28 Maiman M, Fruchter RG, Serur E, et al. Recurrent cervical intraepithelial neoplasia in human immunodeficiency virus-positive women. Obstet Gynecol 1993;82:170-4. 